\title{
METABÓLITOS SECUNDÁRIOS DE ESPÉCIES DE ANACARDIACEAE
}

Suzimone de J. Correia

Departamento de Química e Exatas, Universidade Estadual do Sudoeste da Bahia, 45200-000 Jequié - BA, Brasil

Juceni P. David

Faculdade de Farmácia, Universidade Federal da Bahia, 40170-290 Salvador - BA, Brasil

Jorge M. David*

Instituto de Química, Universidade Federal da Bahia, Campus Univ. Ondina, 40170-290 Salvador - BA, Brasil

Recebido em 18/5/05; aceito em 14/11/05; publicado na web 13/7/06

\begin{abstract}
SECUNDARY METABOLITES FROM SPECIES OF ANACARDIACEAE. This review describes some aspects of the family Anacardiaceae dealing with the presence and distribution of secondary metabolites in the main genera of this family and their biological activities. It reports the occurrence of different natural compounds present in their species with special emphasis on phenolic lipids, flavonoids and triterpenes that are typical metabolites of this family.
\end{abstract}

Keywords: Anacardiaceae; phenolic lipids; flavonoids.

\section{INTRODUÇÃO}

Anacardiaceae é uma família constituída por cerca de 76 gêneros e 600 espécies. Seus gêneros são subdivididos em cinco tribos (Anacardieae, Dobineae, Rhoeae, Semecarpeae e Spondiadeae). Cerca de $25 \%$ dos gêneros dessa família são conhecidos como tóxicos e causadores de dermatite de contato severa. De modo geral, as espécies venenosas desta família estão restritas às tribos Anacardieae, Rhoeae e Semecarpeae ${ }^{1,2}$. A dermatite de contato provocada por essas plantas é atribuída principalmente a compostos fenólicos e catecólicos ou a mistura destas substâncias, denominados lipídios fenólicos. Estas substâncias podem estar presentes em diferentes partes do material vegetal, ocorrendo principalmente em espécies do gênero $R h u s^{3,4}$. Nos últimos anos, a origem dos lipídios fenólicos e derivados também foi objeto de investigação; além disso, espécies da família Anacardiaceae têm se mostrado bastante promissoras na busca de substâncias bioativas. Do ponto de vista químico, os gêneros mais estudados nesta família são Mangifera, Rhus (Toxicodendron), Anacardium, Spondias, Lannea, Semecarpus, Schinus, Pistacia, Lithraea, Tapirira e Melanorrhoea. Mangifera, Rhus e Anacardium destacam-se pelo número de investigações relativas à composição química de suas espécies e atividades biológicas de seus extratos e metabólitos. Os estudos destas espécies possibilitaram verificar a ocorrência de flavonóides, terpenos, esteróides, xantonas e, principalmente, dos lipídios fenólicos e derivados. Destaca-se que entre os flavonóides, os biflavonóides são os mais freqüentes.

\section{O GÊNERO Mangifera}

São conhecidas 58 espécies do gênero Mangifera distribuídas em dois subgêneros. O subgênero Mangifera é constituído por quatro secções: Marchandora, Eurantherae, Rawa, Mangifera. Limus é o segundo subgênero, consistindo das secções Deciduae e Perrennis ${ }^{5}$. M. indica (manga comum) é uma espécie muito consumida, cultivada nas regiões tropicais e subtropicais do mundo. É empregada na medicina popular para uma ampla variedade de enfermidades e,

*e-mail: jmdavid@ufba.br na literatura, encontram-se vários trabalhos descrevendo atividades antiviral, antimicrobiana e antiinflamatória. Nos últimos anos, estudos realizados com extratos aquosos das cascas de uma variedade selecionada de $M$. indica resultaram em uma formulação farmacêutica com nome fantasia de Vimang ${ }^{\circledR}$. O principal componente deste extrato é a mangiferina (Figura 1). Porém, além desta substância, o extrato contém também uma mistura de componentes polifenólicos, terpenóides, esteróides, ácidos graxos e microelementos ${ }^{6}$. Encontra-se descrito na literatura que Vimang ${ }^{\circledR}$ apresenta atividades imunoestimulante ${ }^{7}$, antiiflamatória ${ }^{6}$, antioxidante ${ }^{8-10}$, citotóxica e antineoplásica ${ }^{11}$. Estudos realizados com o extrato aquoso das cascas de $M$. indica demonstraram que é um poderoso seqüestrador de radicais hidroxil e ácido hipocloroso, exerce efeito inibitório sobre a peroxidação de fosfolipídios em cérebro de ratos e inibição de danos no DNA ${ }^{8}$. Além destes, apresenta propriedades de proteção contra a produção de espécies reativas de oxigênio, sendo mais ativo que as vitaminas $\mathrm{C}$ e $\mathrm{E}$, mangiferina e $\beta$-caroteno ${ }^{8}$.<smiles>O=c1c2cc(O)c(O)cc2oc2cc(O)c(O[C@H]3O[C@H](CO)[C@@H](O)[C@H](O)[C@H]3O)c(O)c12</smiles>

Figura 1. Estrutura da mangiferina

No entanto, os terpenóides, principalmente os triterpenos, são as substâncias mais representativas do gênero Mangifera. De $M$. indica foram obtidos triterpenos de esqueletos cicloartano, damarano, taraxastano e hopano ${ }^{12}$ (Tabela 1). Além da mangiferina, foram isolados de $M$. indica compostos fenólicos simples com comprovada atividade antioxidante, tais como ácido hidroxi-benzóico, galatos de alquila, ésteres do ácido benzóico, além dos flavonóides catequina e epi-catequina ${ }^{10}$, e de seu látex foram identificados compostos conhecidos como lipídios fenólicos ${ }^{13}$. 


\section{O GÊNERO Rhus}

Este gênero é o maior da família, com cerca de 200 espécies. Estudos fitoquímicos realizados até o presente momento têm demonstrado que as espécies deste gênero são ricas em flavonóides, principalmente biflavonóides (Tabela 2). Dentre estes destacam-se hinokiflavona (95), amentoflavona (96), agathisflavona (97), robustaflavona $(\mathbf{9 8})$, rhusflavanona (113), succedaneaflavanona (115) e rhusflavona $(\mathbf{1 1 6})^{30-32}$. O interesse nestes compostos está relacionado à expressiva atividade biológica apresentada por eles.

Tabela 1. Terpenóides e esteróides no gênero Mangifera e demais espécies de Anacardiaceae

\section{Mangifera indica ${ }^{13,14}$}
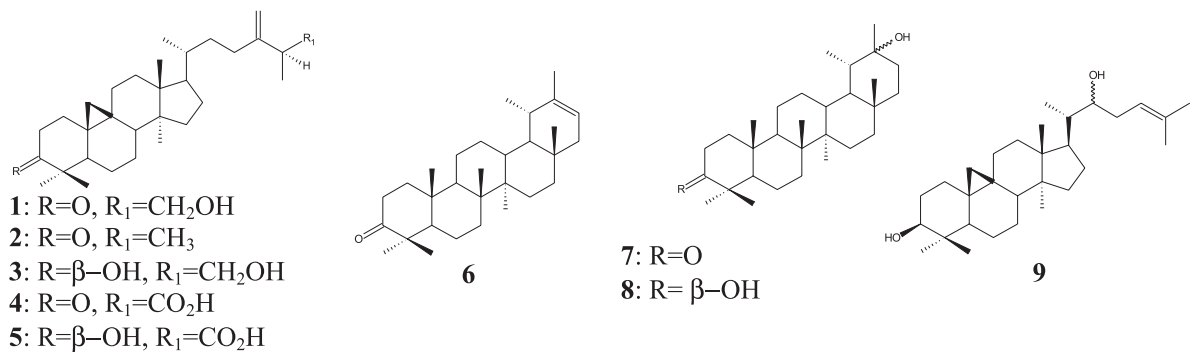<smiles>CCC(C)C(C)CCC1CCC2C3CC(C)C4=CC(=O)CCC4(C)C3CCC12C</smiles>

5: $\mathrm{R}=\beta-\mathrm{OH}, \mathrm{R}_{1}=\mathrm{CO}_{2} \mathrm{H}$<smiles>C=C1CCC2(C)C(C)CCC3C(CCC2C1)CCC1(C)C(C(C)CC=CC(C)C)CCC31</smiles>

11: $\mathrm{R}=\mathrm{O}, \mathrm{R}_{1}=\mathrm{H}$

12: $\mathrm{R}=\beta-\mathrm{OH}$

13: $\mathrm{R}_{1}=\mathrm{OH}$

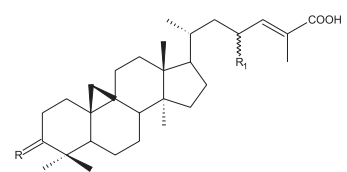

14: $\mathrm{R}=\mathrm{O}, \mathrm{R}_{1}=\mathrm{OH}$

15: $\mathrm{R}=\beta-\mathrm{OH}, \mathrm{R}_{1}=\mathrm{OH}(R)$

16: $\mathrm{R}=\beta-\mathrm{OH}, \mathrm{R}_{1}=\mathrm{OH}(S)$

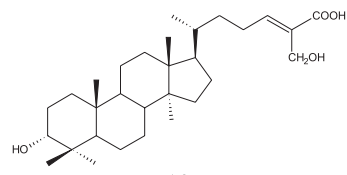

19

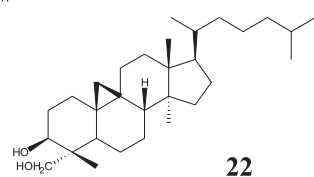

22

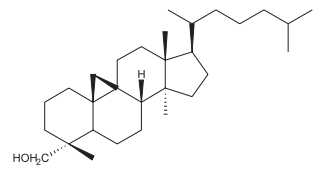

23

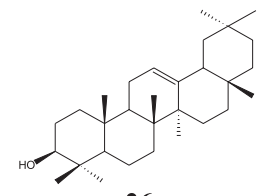

26<smiles>CC1C(=O)C=CC2(C)CCC(C(C)(C)C)CC12</smiles>

24<smiles>CC1CCC2(C)C(C)CCC3C4CCC5(C)CCC(C)(C)CC5C4CCC3C2C1</smiles>

18
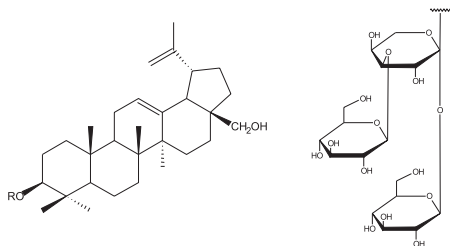

20: $\mathrm{R}=$

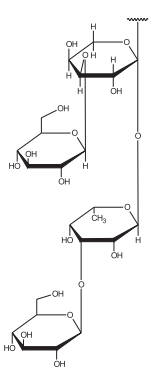

21: $\mathrm{R}=$
18

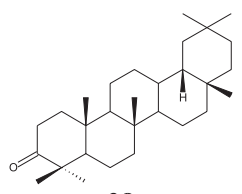

28

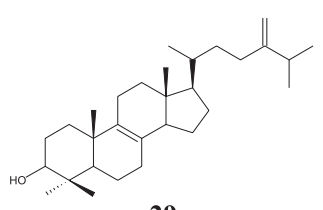

29

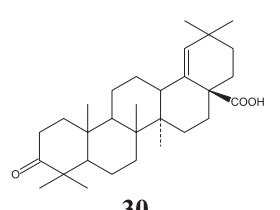

30

Pistacia lentiscus ${ }^{18}$

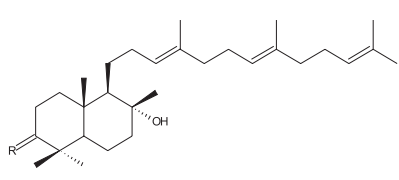

31: $\mathrm{R}=\beta-\mathrm{OH}, \mathrm{H}$

32: $\mathrm{R}=\mathrm{O}$

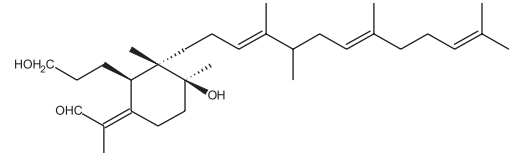

33

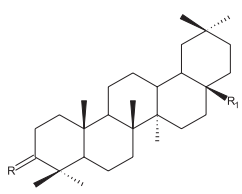

34: $\mathrm{R}=\mathrm{O}, \mathrm{R}_{1}=\mathrm{H}$

35: $\mathrm{R}=\beta-\mathrm{OH}, \mathrm{H} ; \mathrm{R}_{1}=\mathrm{H}$

36: $\mathrm{R}=\mathrm{O}, \mathrm{R}_{1}=\mathrm{CH}_{2} \mathrm{OH}$ 
Tabela 1. continuação

Pistacia lentiscus ${ }^{18}$

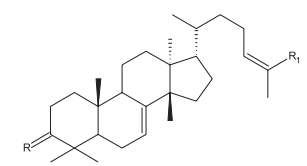

43: $\mathrm{R}=\mathrm{O}, \mathrm{R}_{1}=\mathrm{COOCH}_{3}$

44: $\mathrm{R}=\alpha-\mathrm{OAc}$;

$\mathrm{R}_{1}=\mathrm{COOCH}_{3}$

45: $\mathrm{R}=\beta-\mathrm{OH}$

$\mathrm{R}_{1}=\mathrm{COOCH}_{3}$

46: $\mathrm{R}=\alpha-\mathrm{OH}$,

$\mathrm{R}_{1}=\mathrm{COOCH}_{3}$,

47: $\mathrm{R}=\beta-\mathrm{OH}, \mathrm{R}_{1}=\mathrm{CH}_{2} \mathrm{OH}$

48: $\mathrm{R}=\mathrm{O}, \mathrm{R}_{1}=\mathrm{CHO}$

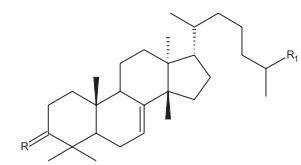

49: $\mathrm{R}=\mathrm{O}, \mathrm{R}_{1}=\mathrm{COOCH}_{3}$ 50: $\mathrm{R}=\alpha-\mathrm{OAc}$, $\mathrm{R}_{1}=\mathrm{COOCH}_{3}$

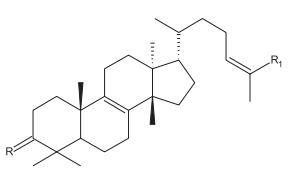

51: $\mathrm{R}=\mathrm{O}, \mathrm{R}_{1}=\mathrm{COOCH}_{3}$

52: $\mathrm{R}=\alpha-\mathrm{OAc}, \mathrm{R}_{1}=\mathrm{COOCH}_{3}$

53: $\mathrm{R}=\beta \mathrm{OH}, \mathrm{R}_{1}=\mathrm{CH}_{3}$

54: $\mathrm{R}=\mathrm{O}, \mathrm{R}_{1}=\mathrm{CH}_{2} \mathrm{OH}$

55: $\mathrm{R}=\beta-\mathrm{OH}, \mathrm{R}_{1}=\mathrm{CH}_{2} \mathrm{OH}$

56: $\mathrm{R}=\mathrm{O}, \mathrm{R}_{1}=\mathrm{CH}_{3}$

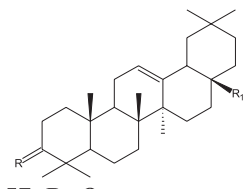

57: $\mathrm{R}=\mathrm{O}$,

$\mathrm{R}_{1}=\mathrm{COOCH}_{3}$

58: $\mathrm{R}=\mathrm{O}, \mathrm{R}_{1}=\mathrm{CH}_{3}$

59: $\mathrm{R}=\mathrm{O}, \mathrm{R}_{1}=\mathrm{CHO}$

60: $\mathrm{R}=\beta-\mathrm{OH}$,

$\mathrm{R}_{1}=\mathrm{CHO}$

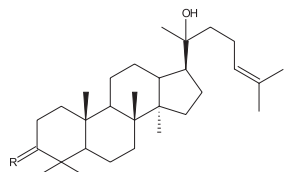

61: $\mathrm{R}=\mathrm{O}$

62: $\mathrm{R}=\beta-\mathrm{OH}$

\section{Pistacia terebinthus ${ }^{20}$}

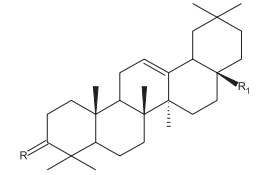

63: $\mathrm{R}=\mathrm{O}, \mathrm{R}_{1}=\mathrm{CHO}$

64: $\mathrm{R}=\beta-\mathrm{OH}, \mathrm{R}_{1}=\mathrm{CH}_{2} \mathrm{OH}$

65: $\mathrm{R}=\beta-\mathrm{OH}, \mathrm{R}_{1}=\mathrm{CHO}$

66: $\mathrm{R}=\mathrm{O}, \mathrm{R}_{1}=\mathrm{COOH}$

67: $\mathrm{R}=\mathrm{O}, \mathrm{R}_{1}=\mathrm{CH}_{2} \mathrm{OH}$

68: $\mathrm{R}=\beta-\mathrm{OH}, \mathrm{R}_{1}=\mathrm{COOH}$

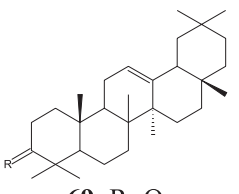

69: $\mathrm{R}=\mathrm{O}$

26: $\mathrm{R}=\mathrm{H}, \beta-\mathrm{OH}$

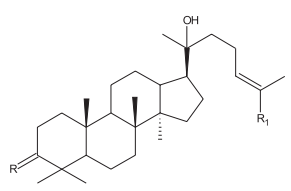

70: $\mathrm{R}=\mathrm{O}, \mathrm{R}_{1}=\mathrm{COOH}$

71: $\mathrm{R}=-\mathrm{OH}, \mathrm{R}_{1}=\mathrm{COOH}$

72: $\mathrm{R}=\mathrm{O}, \mathrm{R}_{1}=\mathrm{CHO}$

73: $\mathrm{R}=\beta-\mathrm{OH}, \mathrm{R}_{1}=\mathrm{CH}_{2} \mathrm{OH}$

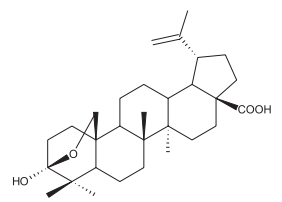

74

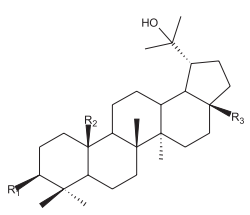

$75 \mathrm{R}_{1}=\beta-\mathrm{OH}, \mathrm{R}_{2}=\mathrm{CH}_{2} \mathrm{OH}, \mathrm{R}_{3}=\mathrm{CH}_{3}$

76: $\mathrm{R}_{1}=\beta-\mathrm{OH}, \mathrm{R}_{2}=\mathrm{CH}_{3}, \mathrm{R}_{3}=\mathrm{CH}_{3}$

$77 \mathrm{R}_{1}=\mathrm{O}, \mathrm{R}_{2}=\mathrm{CH}_{3}, \mathrm{R}_{3}=\mathrm{CH}_{3}$

78: $\mathrm{R}_{1}=\mathrm{O}, \mathrm{R}_{2}=\mathrm{CH}_{3}, \mathrm{R}_{3}=\mathrm{CH}_{2} \mathrm{OH}$

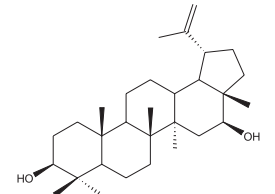

79

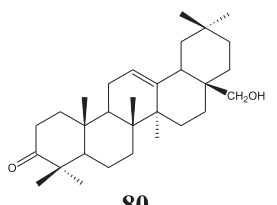

80

Schinus terebenthifolius ${ }^{25}$

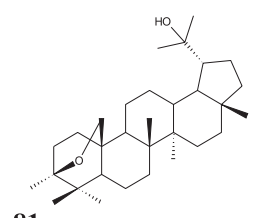

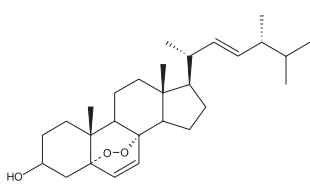

82

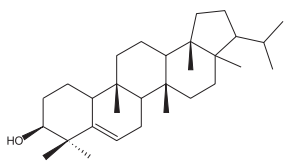

83

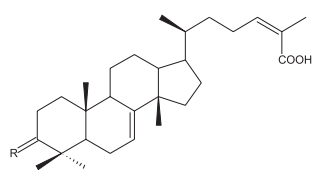

84: $\mathrm{R}=\mathrm{O}$

85: $\mathrm{R}=\mathrm{OH}$ 
Tabela 1. continuação

\section{Schinus molle ${ }^{26}$}

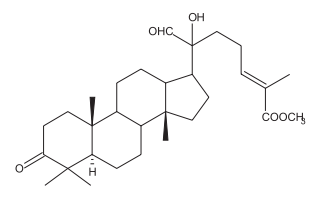

86

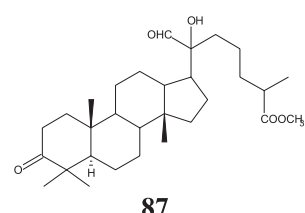

87

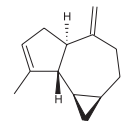

88

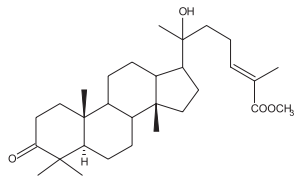

89

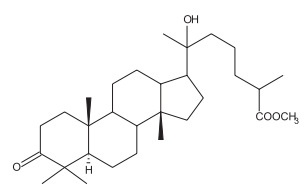

90

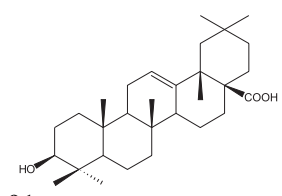

91

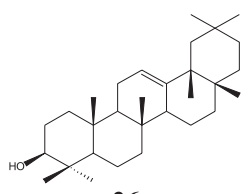

26

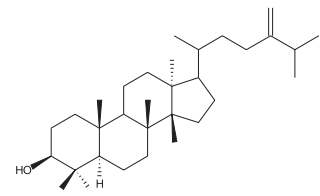

92

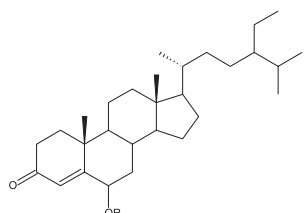

10: $\mathrm{R}=\mathrm{H}$

93: $\mathrm{R}=\mathrm{OH}$

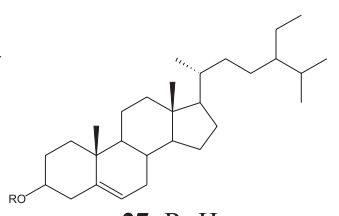

27: $\mathrm{R}=\mathrm{H}$

94: $\mathrm{R}=$ glicosil

Tabela 2. Flavonóides do gênero Rhus e demais espécies de Anacardiaceae

\section{Rhus alata $^{37}$}

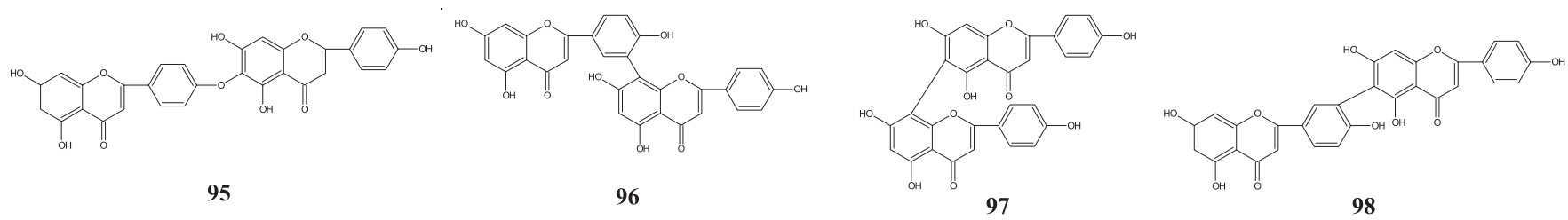

Rhus corlaria ${ }^{38}$

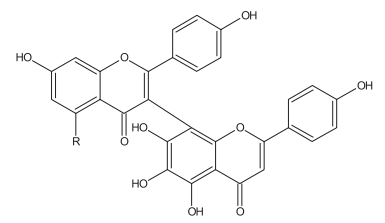

99

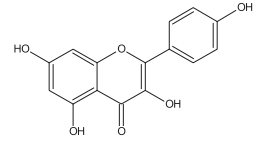

100

Rhus salicifolia ${ }^{39}$

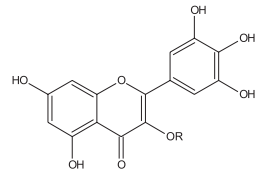

101

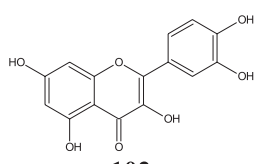

102

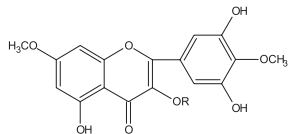

103: $\mathrm{R}=\mathrm{H}$

104: $\mathrm{R}=$ Galactosil

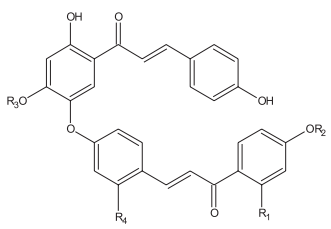

105: $\mathrm{R}_{1}=\mathrm{R}_{4}=\mathrm{H}, \mathrm{R}_{2}=\mathrm{R}_{3}=\mathrm{R}_{5}=\mathrm{CH}_{3}$

106: $\mathrm{R}_{1}=\mathrm{OH}, \mathrm{R}_{2}=\mathrm{R}_{3}=\mathrm{CH}_{3}, \mathrm{R}_{4}=\mathrm{R}_{5}=\mathrm{H}$

107: $\mathrm{R}_{1}=\mathrm{OH}, \mathrm{R}_{2}=\mathrm{R}_{3}=\mathrm{R}_{4}=\mathrm{R}_{5}=\mathrm{H}$

108: $\mathrm{R}_{1}=\mathrm{OH}, \mathrm{R}_{3}=\mathrm{CH}_{3}, \mathrm{R}_{2}=\mathrm{R}_{4}=\mathrm{R}_{5}=\mathrm{H}$

109: $\mathrm{R}_{1}=\mathrm{OH}, \mathrm{R}_{2}=\mathrm{CH}_{3}, \mathrm{R}_{3}=\mathrm{R}_{4}=\mathrm{R}_{5}=\mathrm{H}$

110: $\mathrm{R}_{1}=\mathrm{R}_{2}=\mathrm{R}_{5}=\mathrm{H}, \mathrm{R}_{3}=\mathrm{CH}_{3}, \mathrm{R}_{4}=\mathrm{OH}$

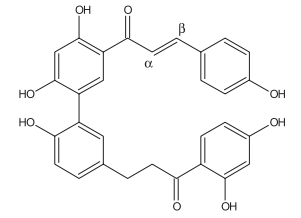

111

112: $\Delta^{\alpha \beta}$

Rhus succedanea $a^{30-33}$

95

96

97

98

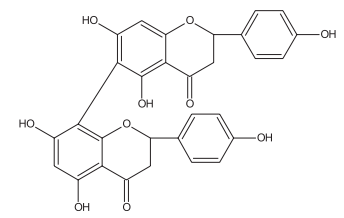

113

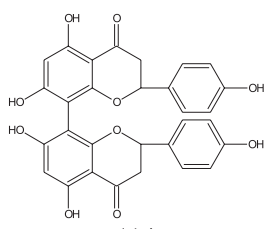

114

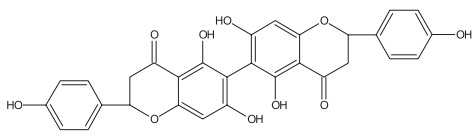

115

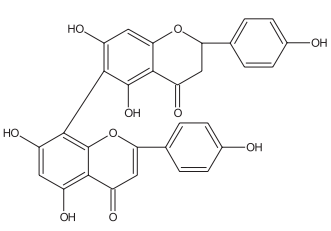

116 
Vol. 29, No. 6

Metabólitos secundários de espécies de Anacardiaceae

1291

Tabela 2. continuação

Rhus retinorrhoea ${ }^{35}$

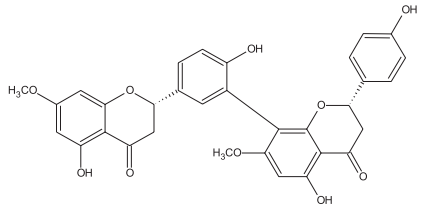

117

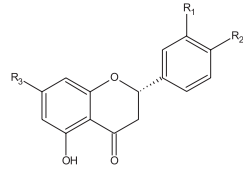

118: $\mathrm{R}_{1}=\mathrm{H}, \mathrm{R}_{2}=\mathrm{OH}, \mathrm{R}_{3}=\mathrm{OMe}$

119: $\mathrm{R}_{1}=\mathrm{R}_{2}=\mathrm{R}_{3}=\mathrm{OH}$

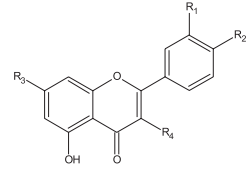

120: $\mathrm{R}_{1}=\mathrm{R}_{3}=\mathrm{OMe}, \mathrm{R}_{2}=\mathrm{R}_{4}=\mathrm{OH}$

121: $\mathrm{R}_{1}=\mathrm{R}_{4}=\mathrm{H}, \mathrm{R}_{2}=\mathrm{OH}, \mathrm{R}_{3}=\mathrm{OMe}$

122: $\mathrm{R}_{1}=\mathrm{R}_{2}=\mathrm{OH}, \mathrm{R}_{3}=\mathrm{OMe}, \mathrm{R}_{4}=\mathrm{H}$

Anacardium occidentale $^{4,42,43}$

Buchanania lazan ${ }^{44}$
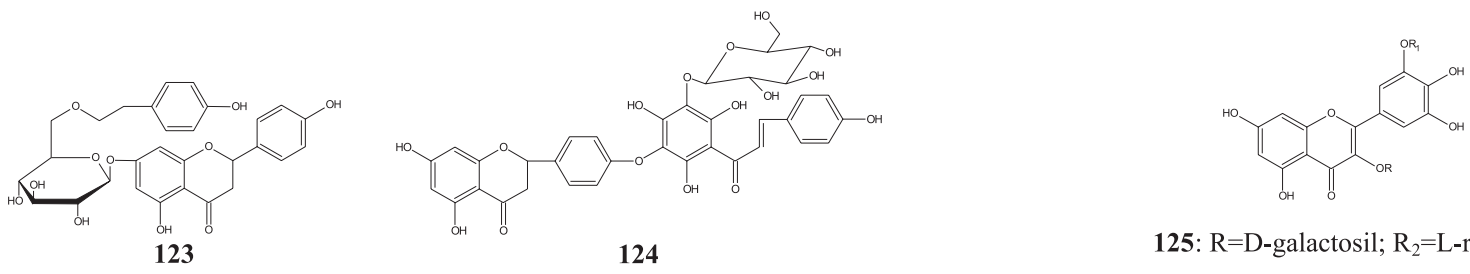

124

125: $\mathrm{R}=\mathrm{D}$-galactosil; $\mathrm{R}_{2}=\mathrm{L}$-ramnosil

Campnosperma panamense ${ }^{45}$

Cloerospondias axiliaris $^{46}$
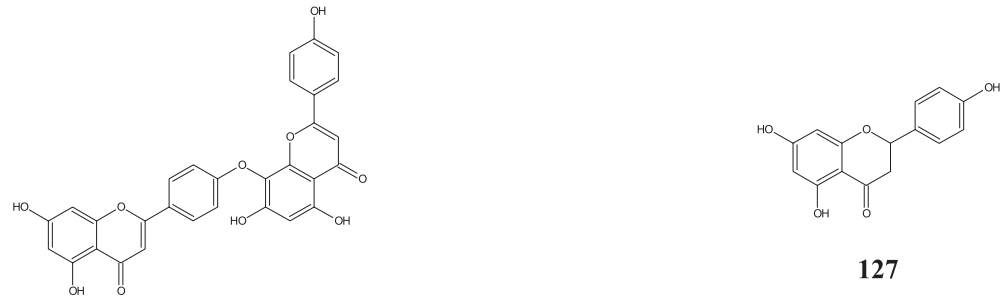

126

127

Cotinus coggygria ${ }^{47}$

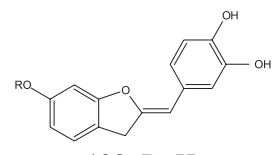

128: $\mathrm{R}=\mathrm{H}$

129: $\mathrm{R}=$ glicosil
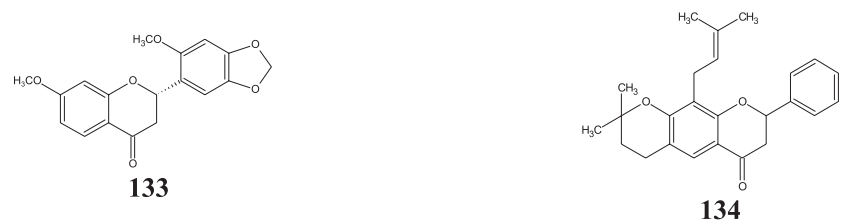

Lannea acida ${ }^{48}$

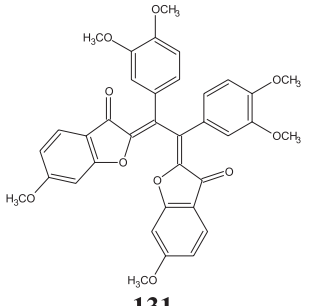

131

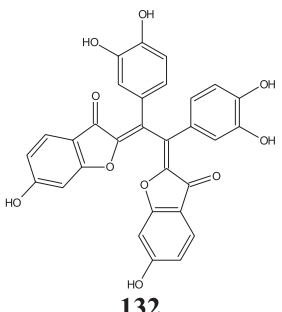

132

Lannea coromandelica ${ }^{49}$<smiles>CCOc1cc2c(cc1C)OC(c1ccc(C)c(C)c1)C(C)C2=O</smiles>

135: $\mathrm{R}_{1}=\mathrm{OH}, \mathrm{R}_{2}=\mathrm{OCH}_{3}, \mathrm{R}_{3}=\mathrm{OH}$, $\mathrm{R}_{4}=\mathrm{OCH}_{3}, \mathrm{R} 5=3 S-\mathrm{OH}$

136: $\mathrm{R}_{1}=\mathrm{OCH}_{3}, \mathrm{R}_{2}=\mathrm{OCH}_{3}, \mathrm{R}_{3}=\mathrm{H}$, $\mathrm{R}_{4}=\mathrm{OCH}_{3}, \mathrm{R} 5=3 R-\mathrm{OH}$ 137: $\mathrm{R}_{1}=\mathrm{OH}, \mathrm{R}_{2}=\mathrm{OCH}_{3}, \mathrm{R}_{3}=\mathrm{OH}$, $\mathrm{R}_{4}=\mathrm{OCH}_{3}, \mathrm{R} 5=3 \mathrm{R}-\mathrm{OH}$

138: $\mathrm{R}_{1}=\mathrm{OH}, \mathrm{R}_{2}=\mathrm{OCH}_{3}, \mathrm{R}_{3}=\mathrm{H}$, $\mathrm{R}_{4}=\mathrm{OCH}_{3} \mathrm{R} 5=3 R-\mathrm{OH}$ 139: $\mathrm{R}_{1}=\mathrm{OH}, \mathrm{R}_{2}=\mathrm{OH}, \mathrm{R}_{3}=\mathrm{OH}$, $\mathrm{R}_{4}=\mathrm{OCH}_{3} \mathrm{R} 5=3 R-\mathrm{OH}$<smiles>O=c1c(O)c(-c2ccc(O)c(O)c2)oc2cc(O)cc(O)c12</smiles>

102: $\mathrm{R}=\mathrm{H}$

140: $\mathrm{R}=$ arabinosil 
Tabela 2. continuação

\section{Mangifera indica ${ }^{13,14}$}<smiles>Oc1cc(O)c2c(c1)O[C@H](c1ccc(O)c(O)c1)[C@H](O)C2</smiles>

141: $\mathrm{R}=3 R-\mathrm{OH}$

142: $\mathrm{R}=3 S-\mathrm{OH}$<smiles>OC1=C(c2ccc(O)c(Br)c2)Oc2cc(Br)cc(O)c2C1</smiles>

100: $\mathrm{R} 1=\mathrm{H}, \mathrm{R}_{2}=\mathrm{OH}, \mathrm{R}_{3}=\mathrm{H}$

102: $\mathrm{R} 1=\mathrm{OH}, \mathrm{R}_{2}=\mathrm{OH}, \mathrm{R}_{3}=\mathrm{H}$

143: $\mathrm{R}_{1}=\mathrm{OH}, \mathrm{R}_{2}=\mathrm{OCH}_{3}, \mathrm{R}_{3}=$ glicosil

Myracrodruon urundeuva ${ }^{50}$

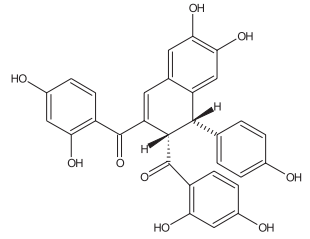

144

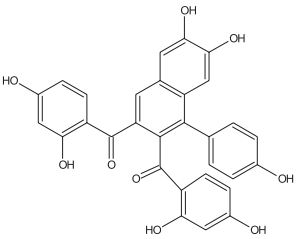

145

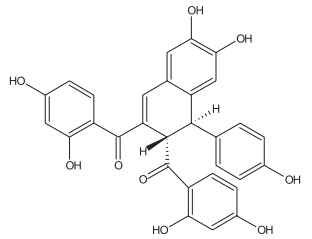

146

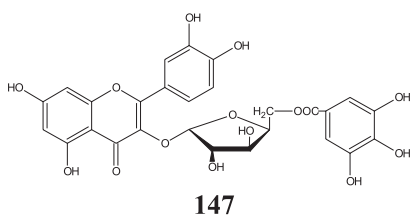

147

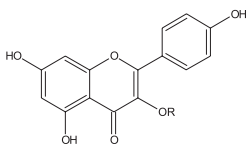

150: $\mathrm{R}=(6 "$ " -galoil $)$-glicose,

151: $\mathrm{R}=$ ramnosil

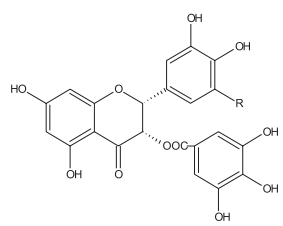

148: $\mathrm{R}=\mathrm{H} ; 1$ 149: $\mathrm{R}=\mathrm{OH}$

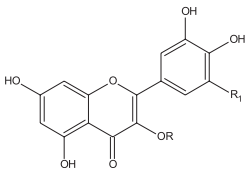

152: $\mathrm{R}=\left(6\right.$ "' -galoil)-glicosil, $\mathrm{R}_{1}=\mathrm{H}$

153: $R=(6 "$ " -galoil $)$-galactosil, $R_{1}=H$

154: $R=$ ramnosil, $R_{1}=H$

155: $\mathrm{R}=$ glicosil, $\mathrm{R}_{1}=\mathrm{H}$

156: $\mathrm{R}=$ ramnosil, $\mathrm{R}_{1}=\mathrm{OH}$
141

Semecarpus prainii ${ }^{53}$

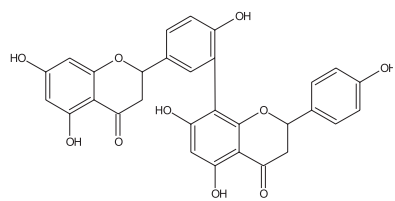

157

Semecarpus anacardium ${ }^{54}$

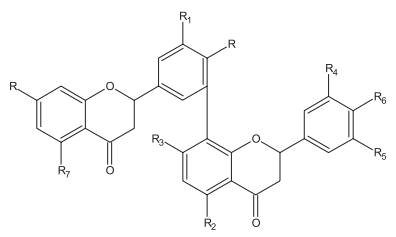

158: $\mathrm{R}=\mathrm{R}_{2}=\mathrm{R}_{4}=\mathrm{R}_{6}=\mathrm{R}_{7}=\mathrm{OH}, \mathrm{R}_{1}=\mathrm{R}_{3}=\mathrm{R}_{5}=\mathrm{H}$

159: $\mathrm{R}=\mathrm{R}_{2}=\mathrm{R}_{4}=\mathrm{R}_{6}=\mathrm{R}_{7}=\mathrm{OH}, \mathrm{R}_{1}=\mathrm{R}_{3}=\mathrm{R}_{5}=\mathrm{H}$

160: $\mathrm{R}=\mathrm{R}_{3}=\mathrm{R}_{6}=\mathrm{R}_{7}=\mathrm{OH}, \mathrm{R}_{1}=\mathrm{R}_{2}=\mathrm{R}_{4}=\mathrm{R}_{5}=\mathrm{H}$

161: $\mathrm{R}=\mathrm{R}_{3}=\mathrm{R}_{4}=\mathrm{R}_{5}=\mathrm{R}_{6}=\mathrm{R}_{7}=\mathrm{OH}, \mathrm{R}_{1}=\mathrm{R}_{2}=\mathrm{H}$

162: $\mathrm{R}=\mathrm{R}_{1}=\mathrm{R}_{3}=\mathrm{R}_{4}=\mathrm{R}_{5}=\mathrm{R}_{6}=\mathrm{R}_{7}=\mathrm{OH}, \mathrm{R}_{2}=\mathrm{H}$

163: $\mathrm{R}=\mathrm{R}_{2}=\mathrm{R}_{3}=\mathrm{R}_{4}=\mathrm{R}_{6}=\mathrm{R}_{7}=\mathrm{OH}, \mathrm{R}_{1}=\mathrm{R}_{5}=\mathrm{H}$

164: $\mathrm{R}=\mathrm{R}_{1}=\mathrm{R}_{2}=\mathrm{R}_{3}=\mathrm{R}_{4}=\mathrm{R}_{5}=\mathrm{OCH}_{3}, \mathrm{R}_{6}=\mathrm{R}_{2}=\mathrm{OH}, \mathrm{R}_{7}=\mathrm{H}$

165: $\mathrm{R}=\mathrm{R}_{4}=\mathrm{R}_{6}=\mathrm{OCH}_{3}, \mathrm{R}_{1}=\mathrm{R}_{3}=\mathrm{OH}, \mathrm{R}_{5}=\mathrm{R}_{7}=\mathrm{R}_{2}=\mathrm{H}$

Hinokiflavona (95), agathisflavona (97) e robustaflavona (98), por ex., atuam na replicação do vírus HIV pela inibição da enzima trancriptase reversa (HIV-1-RT) ${ }^{33}$. Quando comparada com outros 65 flavonóides, a hinokiflavona (17) mostrou-se mais ativa na inibição da atividade pró-coagulante de monócitos humanos aderentes, estimulada por endotoxina e interleucina- $1 \beta^{34}$. Outros biflavo- nóides de Rhus apresentaram atividades biológicas importantes, como antimalárica ${ }^{35}$, antiviral ${ }^{36}$ e citotóxica ${ }^{31}$.

A seiva de espécies deste gênero é aproveitada há mais de 5000 anos na China na produção das conhecidas lacas orientais. A espécie mais apreciada para esse fim é $R$. vernicifera. Porém, $R$. succedanea, usada no Vietnã e Formosa, e espécies de outros gêne- 
ros da família como Melanorrhoea (M. usitate) também são aproveitadas com o mesmo propósito ${ }^{55}$. Na medicina tradicional, algumas espécies do gênero Rhus são usadas como antimicrobianos ou mesmo por suas propriedades citotóxica e inseticida ${ }^{35}$. Espécies deste gênero também são conhecidas por provocarem dermatite alérgica de contato (DAC) muito severa, contraída por manuseio ou ingestão de partes das plantas. Na América do Norte, admite-se que $50-60 \%$ dos americanos sofrem desta dermatite provocada por $R$. radicans ("poison ivy"), $R$. diversibolum ("poison oak"), $R$. vernix e $R$. verniciflua ${ }^{57}$. Estas espécies possuem uma oleoresina conhecida no Ocidente como urushiol. No Japão, a resina conhecida como kiurushi é constituída da seiva de $R$. vernicifera. As resinas são formadas por mistura de $n$-alquilcatecóis com cadeia lateral de 15 e 17 carbonos sendo que os 3- $n$-alquenilcatecóis são mais frequientes que os 4-alquilcatecóis. Em outras espécies de Rhus foram descritas as ocorrências de $n$-alquifenóis e $n$-alquil-hidroquinonas ${ }^{56}$.

Estudos realizados com urushiol isolado de espécies de Rhus mostraram que os verdadeiros antígenos envolvidos na dermatite alérgica são quinonas produzidas a partir da oxidação dos alquilcatecóis, haja vista que estas, além de serem capazes de reagir com os centros nucleofílicos das proteínas, são absorvidas pelo sistema imunológico ${ }^{57}$.

\section{O GÊNERO Anacardium}

O gênero Anacardium possui 11 espécies descritas e $A$. occidentale é seu representante mais conhecido. O caju ( $A$. occidentale), além de ser muito conhecido pelo sabor e valor nutricional de suas amêndoas e pendúculos (pseudofruto), é uma cultura de grande importância sócio-econômica para o Brasil. A amêndoa e o líquido extraído da casca da castanha de caju (CNSL ou LCC) são produtos de exportação ${ }^{58}$.

Os ácidos anacárdicos, juntamente com os alquilresorcinóis, constituem cerca de $90 \%$ da composição do CNSL e apresentam propriedades tóxicas e irritantes. Porém, no pseudofruto, a proporção das substâncias fenólicas é muito baixa ${ }^{59}$. Além dos ácidos anacárdicos, os 1,3-diidroxi-5-alquilbenzenos, conhecidos como lipídios resorcinólicos (cardol), também ocorrem naturalmente em pequenas proporções não só em $A$. occidentale, como também em outras espécies da família. A ocorrência destas substâncias e seus derivados tem sido verificada também em um número crescente de plantas de outras famílias e microorganismos ${ }^{60,61}$. Alquilresorcinóis podem ser encontrados em cereais ${ }^{62}$, como centeio, cevada, trigo, triticale, algas, e sob condições fisiológicas especiais foi encontrado na bactéria Azotobacter vinelandii ${ }^{60}$. Os ácidos anacárdicos são uma mistura de ácidos 6-alquil-salicílico, onde os grupos alquílicos variam tanto no comprimento da cadeia como no grau de insaturação das mesmas, sendo mais freqüentes cadeias mono, di ou triinsaturadas ${ }^{40}$. Devido à termolabilidade do grupo carboxílico eles são prontamente descarboxilados durante o processamento e, assim, tendem a se converter em cardanol, de forma que o principal componente do CNSL é o cardol (mistura dos homólogos insaturados do 5-n-pentadecil resorcinol) ${ }^{42}$.

Nos últimos anos foi descoberta uma série de atividades biológicas para os ácidos anacárdicos; além de atividade antitumoral, destaca-se a habilidade em inibir as enzimas tirosinase ${ }^{63}$, prostaglandina sintase e lipooxigenase ${ }^{64}$. Estes ácidos também são conhecidos por suas atividades antiacne ${ }^{43}$, antibacteriana ${ }^{65}$, moluscocida ${ }^{67}$ e antifúngica $^{60}$. Foram testados 16 compostos fenólicos isolados de $A$. occidentale, dentre estes, 4 ácidos anacárdicos exibiram considerável atividade inibitória contra as bactérias Gram-positivas Streptococcus mutans, Brevibacterium ammoniagenes, Staphylococcus aureus, Bacillus subtilis e Propionibacterium acnes.
Deve-se destacar que o comprimento das cadeias alquílicas destes ácidos influi na atividade. Este fato foi confirmado a partir da comparação da atividade destas substâncias com a do ácido salicílico, que também apresentou atividade contra a maioria dos microorganismos mencionados acima. Apesar dos ácidos anacárdicos exibirem um espectro de atividade limitado contra bactérias, as atividades foram consideravelmente maiores que as apresentadas pelo ácido salicílico. O exemplo que reflete a importância da cadeia lateral dos ácidos anacárdicos para atividade foi dada pelo ácido 6-[8-(Z), 11(Z), 14-pentadecatrienil]salicílico. Quando avaliado contra $S$. mutans e $S$. aureus, mostrou-se 2048 e 64 vezes mais efetivo que o ácido salicílico, respectivamente. Isto sugere que a cadeia alquílica exerce papel importante na aumento da atividade ${ }^{65}$. É digno de nota que, recentemente, têm sido desenvolvidos estudos para uso da semente do cajueiro na preparação de vernizes ${ }^{66}$.

\section{LIPÍDIOS FENÓLICOS E DERIVADOS}

Os metabólitos encontrados em espécies de Anacardium, Rhus (n-alquilcatecóis), Mangifera e em muitos outros gêneros da família Anacardiaceae, listados na Tabela 3, são classificados por alguns autores como lipídios não-clássicos, lipídios fenólicos, alquilfenóis ou ainda como fenóis de cadeia longa ${ }^{4,68}$.

O primeiro lipídio fenólico, do tipo lipídio resorcinólico, foi isolado de Ginkgo biloba (Ginkgoaceae) ${ }^{68}$. Posteriormente sua ocorrência foi verificada em outras famílias de plantas superiores, tais como Proteaceae, Myrsinaceae, Primulaceae, Miristicaceae, Iridaceae, Araceae, Asteraceae, Leguminosae e Gramineae. No entanto, Anacardiaceae tem sido sua principal fonte. Inicialmente estas substâncias foram encontradas em frutos e sementes de espécies das referidas famílias e, mais recentemente, sua ocorrência também tem sido observada em tecidos ou órgãos verdes, tais como folhas e caules ${ }^{68}$.

Os lipídios fenólicos e derivados são definidos como produtos naturais não-isoprênicos, que apresentam em sua estrutura grupos aromáticos e alifáticos e exibem ambos os comportamentos, hidrofílico e lipofílico. Normalmente são caracterizados pela presença de grupo fenol ligado a uma cadeia alquílica com número ímpar de carbono. Ocorrem como fenóis simples ${ }^{69}$ ou derivados catecólicos ${ }^{70,71}$, resorcinólicos ${ }^{61,72}$ ou hidroquinônicos ${ }^{73,74}$, sendo os resorcinólicos os mais abundantes na natureza ${ }^{4,68}$.

Além da variação em número e posição relativa dos grupos hidroxílicos no anel aromático, os lipídios fenólicos apresentam cadeias alquílicas com diferentes comprimentos, grau de insaturação e posição das ligações duplas ${ }^{1}$. De modo geral, têm comprimento de cadeia lateral com um número ímpar de átomos de carbono, que varia de $\mathrm{C}_{15}-\mathrm{C}_{29}$. No entanto, os comprimentos mais comuns são $\mathrm{C}_{15}$ e $\mathrm{C}_{17}$. Em cereais, ao contrário das outras fontes, misturas de lipídios fenólicos contêm uma variedade de homólogos com comprimento da cadeia lateral variando de $\mathrm{C}_{15}-\mathrm{C}_{27}{ }^{62}$. Considerando-se as posições das ligações duplas, elas ocorrem mais freqüentemente em $\Delta^{8^{\prime}} \Delta^{11^{\prime}}$ e $\Delta^{14^{4} 4,68}$.

A presença de regiões hidrofílica e hidrofóbica nas estruturas das moléculas dos lipídios fenólicos em geral confere um caráter anfipático, responsável pela elevada afinidade entre estes e as bicamadas lipídicas e membranas biológicas. Esta característica permite que os lipídios fenólicos sejam facilmente incorporados às membranas celulares, provocando mudanças na sua estrutura e propriedades. O efeito de estabilização dos lipídios fenólicos e derivados nas membranas também é resultado da interação dos grupos hidroxílicos do anel aromático com fosfolipídios, por meio de ligações de hidrogênio ${ }^{68}$.

As cadeias alquílicas exercem influência significativa na atividade biológica que pode estar relacionada com o aumento da solu- 
Tabela 3. Lipídios fenólicos e derivados isolados de espécies de Anacardium e demais gêneros de Anacardiaceae

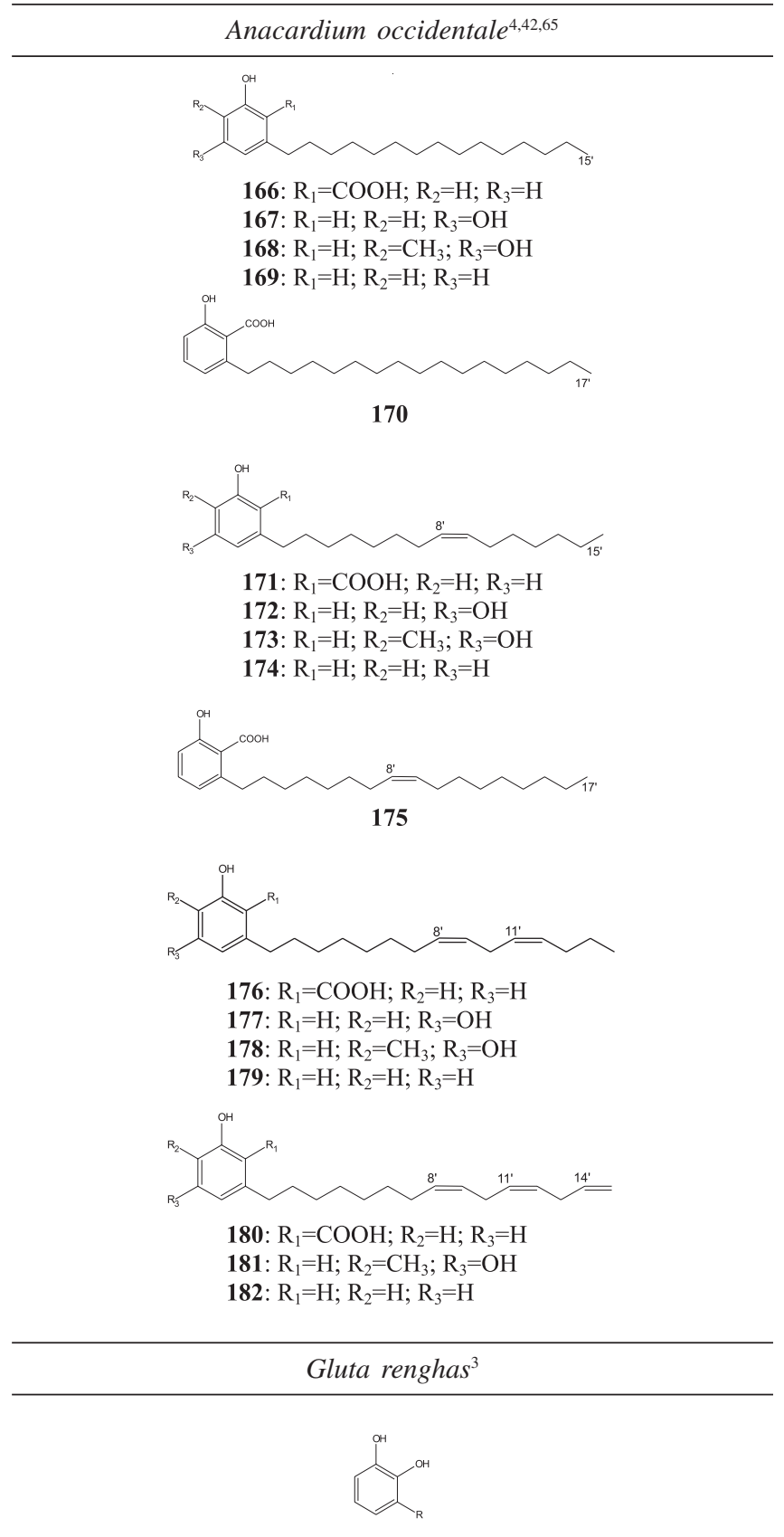

183: $\mathrm{R}=\mathrm{C}_{17} \mathrm{H}_{31}$ *

Lannea edulis ${ }^{77}$

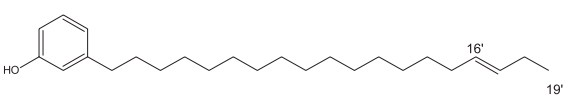

184

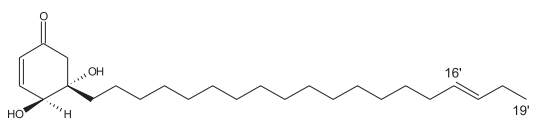

185

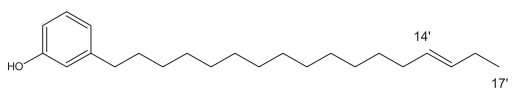

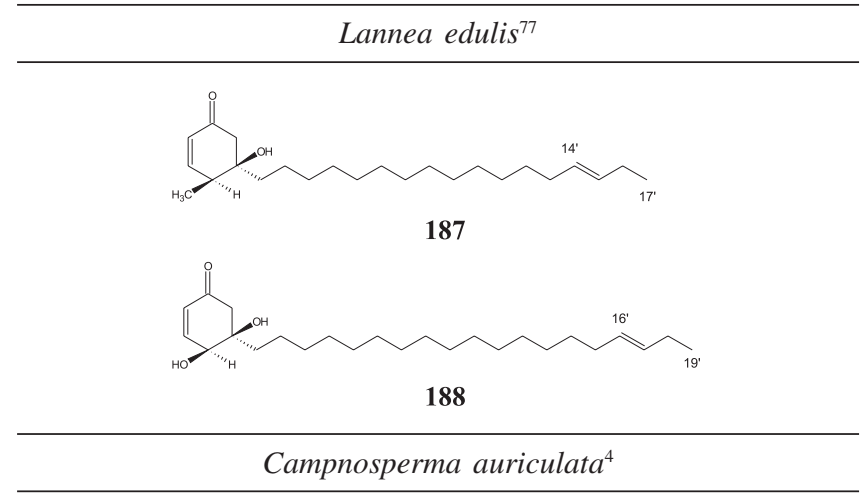

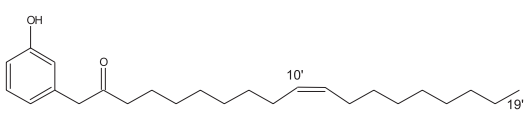

189

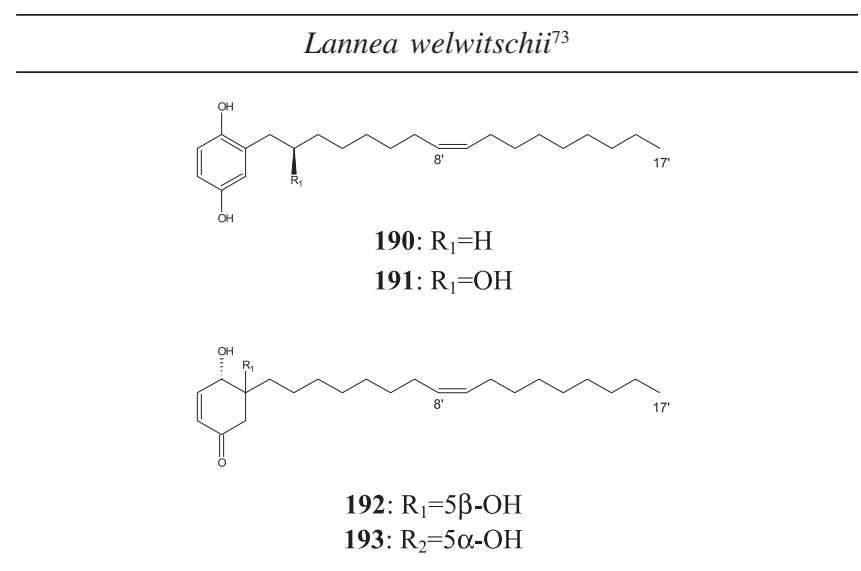

Lithraea caustica ${ }^{70}$

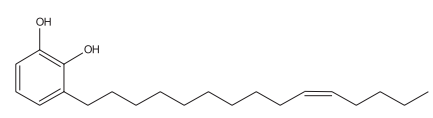

194

Lithraea molleoides ${ }^{78,79}$

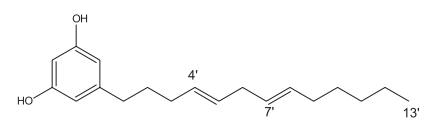

195

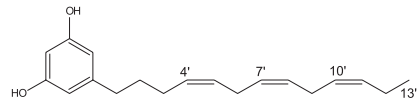

196

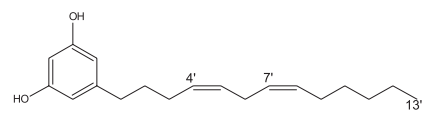

197

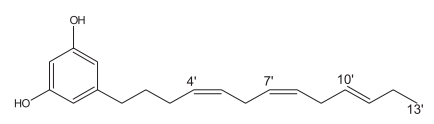

198

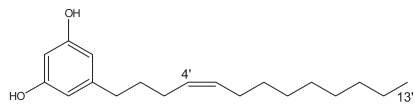

199 


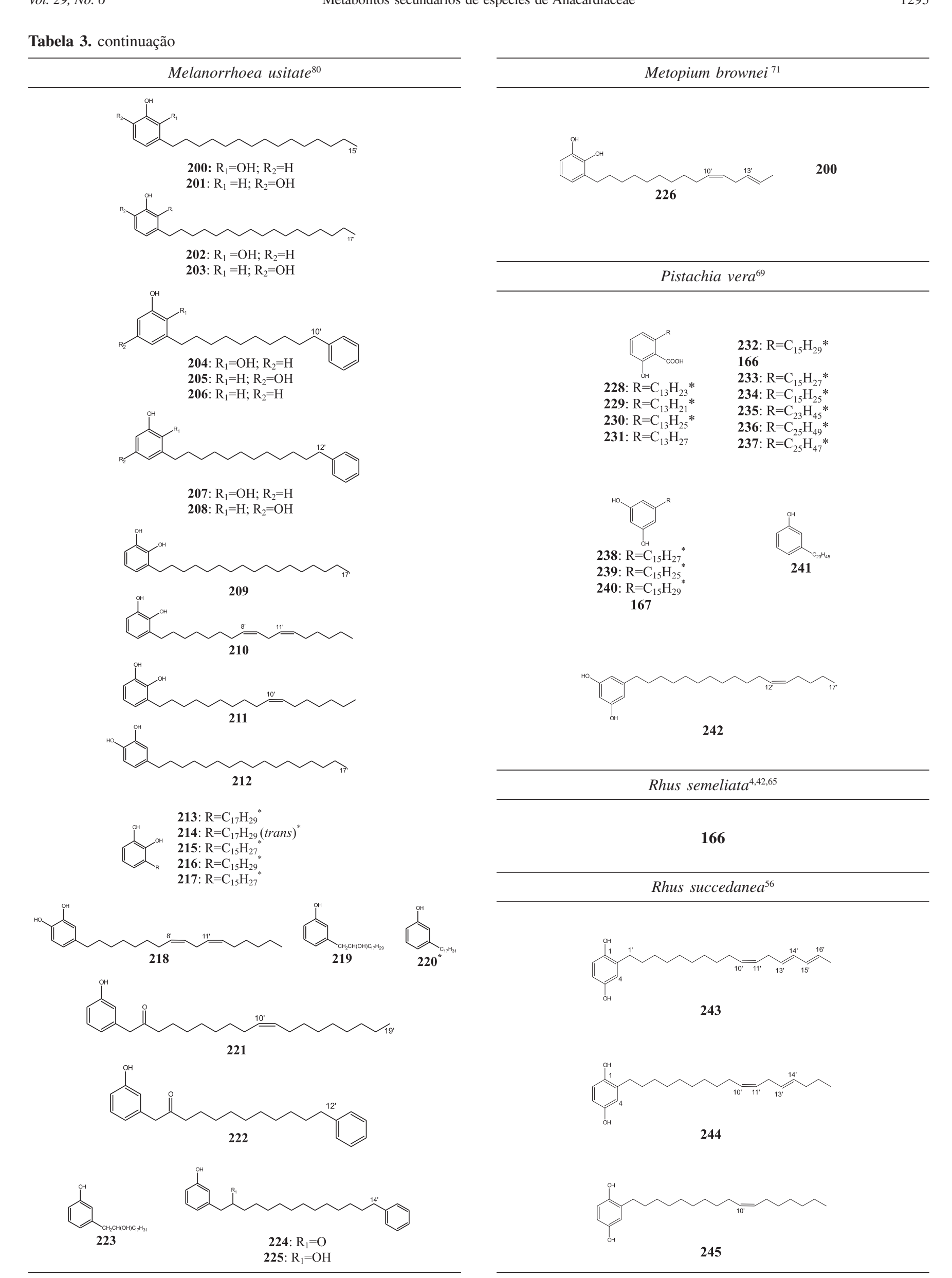


Tabela 3. continuação

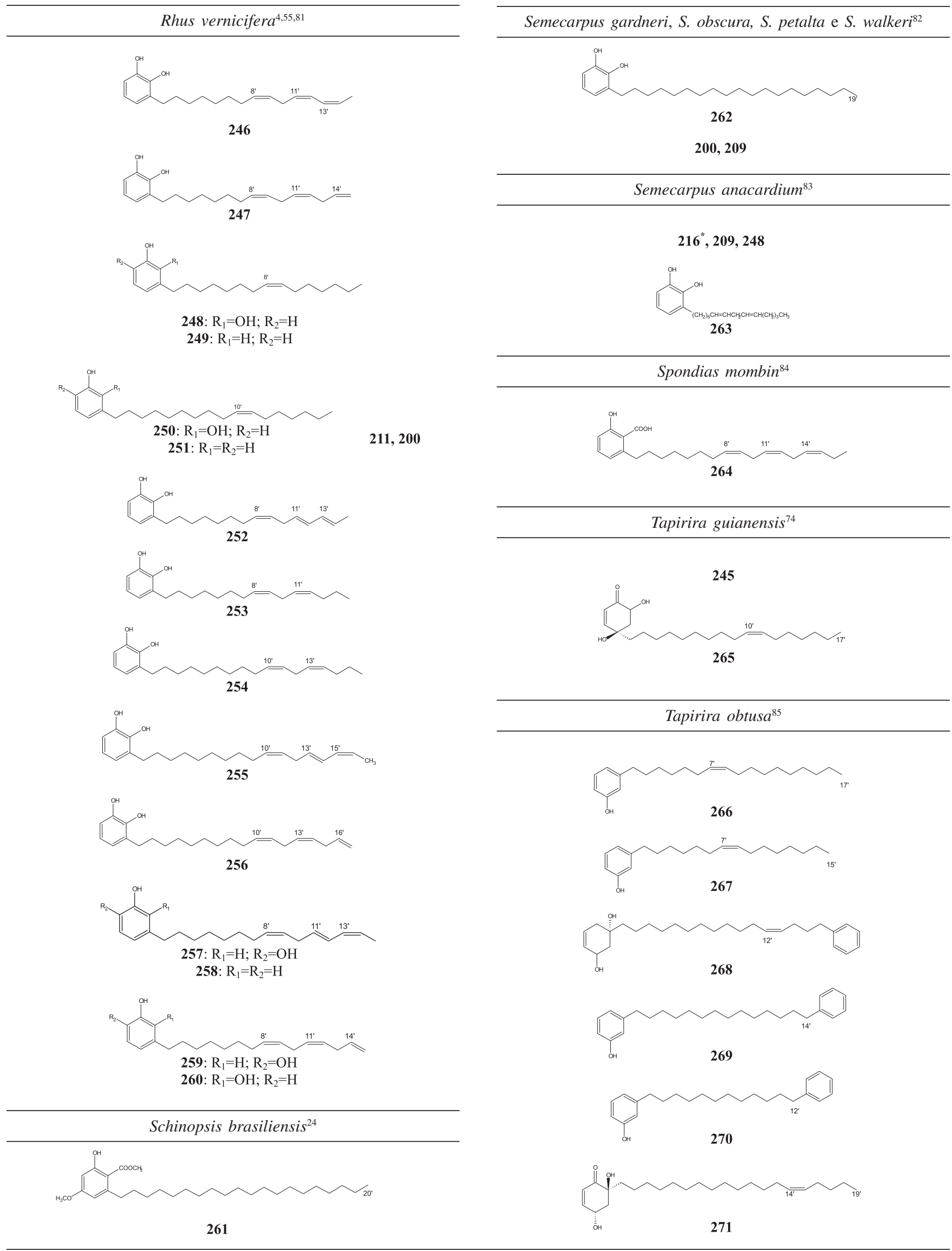

\footnotetext{
* Substâncias em que as posições das ligações duplas não foram determinadas.
} 
bilidade das porções fenólicas nas regiões lipídicas, nas quais é requerida proteção contra degradação biológica ou oxidação química. A influência da cadeia alquílica no mecanismo de proteção é similar ao efeito que é proposto para a cadeia poliprenílica do tocoferol ${ }^{75}$. Observou-se que os alquilresorcinóis, por ex., em concentrações micromolares, comportam-se como antioxidantes ativos, protegendo os ácidos graxos livres e fosfolipídios contra a peroxidação induzida por íon ferro, auto-oxidação de ácidos graxos insaturados e triglicerídeos, assim como da oxidação das membranas biológicas. Estas atividades possivelmente são a razão para a atividade antimutagênica apresentada por estes compostos ${ }^{62}$.

Além das funções biológicas, esses compostos possuem elevada importância como matéria-prima industrial. Os lipídios não clássicos isolados das cascas da castanha do caju (Tabela 3), um subproduto agrícola, constituem uma fonte potencial de monômeros para produção de polímeros. Polímeros derivados do CNSL e cardonol geralmente são preparados pela condensação com agentes eletrofílicos, como formaldeído, pela polimerização da cadeia por meio das insaturações da cadeia lateral usando catálise ácida ou pela funcionalização da hidroxila fenólica e subseqüente oligopolimerização para se conseguirem pré-polímeros funcionalizados ${ }^{76}$. Estes polímeros têm despertado interesse, pois o CNSL advém de uma fonte renovável. Apesar de apresentarem uma distribuição relativamente ampla, a princípio, os lipídeos fenólicos poderiam ser considerados como marcadores quimiotaxonômicos da família, haja visto que ocorrem em vários gêneros da família (Tabela 3).

\section{Biossíntese de lipídios fenólicos}

O pioneirismo no estudo da biossíntese dos lipídios fenólicos pode ser atribuído a Arthur Birch que, em 1952, propôs a participação do acetato na biossíntese do ácido 6-metilsalicílico. A incorporação do [ [14-C]-ácido acético no ácido-6-metilsalicílico foi obtida a partir de Penicillium griseofulvum. Neste estudo foi verificado o padrão de marcação alternado no produto e demonstrado que $\left[{ }^{14} \mathrm{C}-{ }^{18} \mathrm{O}\right]$-ácido acético é incorporado no ácido orselínico (ácido-4hidroxi-6-metilsalicílico) com retenção de marcação. Este fato demonstrou que os átomos de oxigênio do anel são oriundos do grupo carboxílico do acetato. Por outro lado, um dos oxigênios do grupo carboxílico do ácido metilsalicílico é oriundo da hidrólise do éster da coenzima-A por $\mathrm{H}_{2}{ }^{18} \mathrm{O}^{86}$.

A origem das cadeias alquílicas é atribuída aos ácidos graxos de cadeia longa, por ex. o ácido palmitoléico, haja vista que este pode sofrer condensação com três unidades de malonato, que seriam responsáveis pela formação do anel aromático. Esta hipótese foi confirmada a partir de experimentos enzimáticos utilizando possíveis precursores marcados com isótopos radiativos. Por extrapolação concluiu-se que a natureza do complexo enzimático envolvido na formação dos ácidos anacárdicos poderia ser semelhante ao complexo enzimático ácido 6-metilsalicílico sintase, partindo-se da observação que o comprimento das cadeias alquílicas é a principal diferença estrutural entre o ácido-6-metilsalicílico e os ácidos anacárdicos. Com a obtenção do ácido-6-etilsalicílico em um experimento in vitro, quando acetil-CoA foi substituído por propionil-CoA, foi possível concluir que a enzima ácido-6-metilsalicílico sintase participa destas reações e que, a partir de um precursor de cadeia mais longa, os ácidos anacárdicos podem ser obtidos pela mesma rota ${ }^{87}$. Estudos posteriores demonstraram a semelhança entre as enzimas 6metilsalić́lico sintase e ácido graxo sintase. Foi ainda demonstrado que é possível obter a enzima ácido 6-metilsalicílico sintase a partir da derivatização da enzima ácido graxo sintase ${ }^{88}$.

Estudos sobre a participação dos ácidos graxos na biossíntese dos ácidos anacárdicos foram realizados administrando-se estearato, oleato, palmitato e miristato de metila nos tecidos de flores e folhas de Pelargonium xhortorum ${ }^{87}$. Após alimentação com $\left[{ }^{14} \mathrm{C}\right]$-palmitato de metila houve a formação do ácido anacárdico $\mathrm{C}_{22}$ saturado, significando que o precursor administrado à espécie é incorporado antes de sofrer qualquer transformação. Por outro lado, quando foi administrado $\left[{ }^{14} \mathrm{C}\right]$-estearato de metila foram detectados os ácidos anacárdicos saturados $\left(\mathrm{C}_{22}\right.$ e $\left.\mathrm{C}_{24}\right)$ e os ácidos anacárdicos $\mathrm{C}_{22}$ e $\mathrm{C}_{24} \omega$ 5 insaturados. Desse modo, parte do estearato de metila administrado foi incorporado à rota direta de formação dos lipídios fenólicos levando à formação do correspondente ácido anacárdico com cadeia alquílica também saturada. Porém, outra parte do precursor sofre $\beta$-oxidação formando o acetil-CoA que é incorporado a outras rotas de formação de metabólitos, inclusive à rota de formação dos ácidos graxos (Figura 2). Os diferentes ácidos graxos formados pela rota indireta podem ser convertidos nos demais ácidos anacárdicos de cadeia maior, também com padrão de insaturação diferente do esperado para o precursor que fora administrado. Caso o estearato fosse incorporado apenas pela rota direta, somente o $\left[{ }^{14} \mathrm{C}\right]$-ácido anacárdico $\mathrm{C}_{24}$ saturado seria formado. Na possibilidade de que apenas o caminho indireto se processasse, a marcação seria encontrada em todos os ácidos anacárdicos em quantidades proporcionais ao total dos ácidos anacárdicos, situação não observada. Estes resultados estão de acordo tanto com a rota direta quanto com a indireta. No caso $\left[10-{ }^{14} \mathrm{C}\right]$-oleato de metila também se verificou incorporação através de um caminho indireto, sugerindo que a $\beta$-oxidação que leva à rota indireta depende do comprimento da cadeia do precursor ${ }^{87}$.

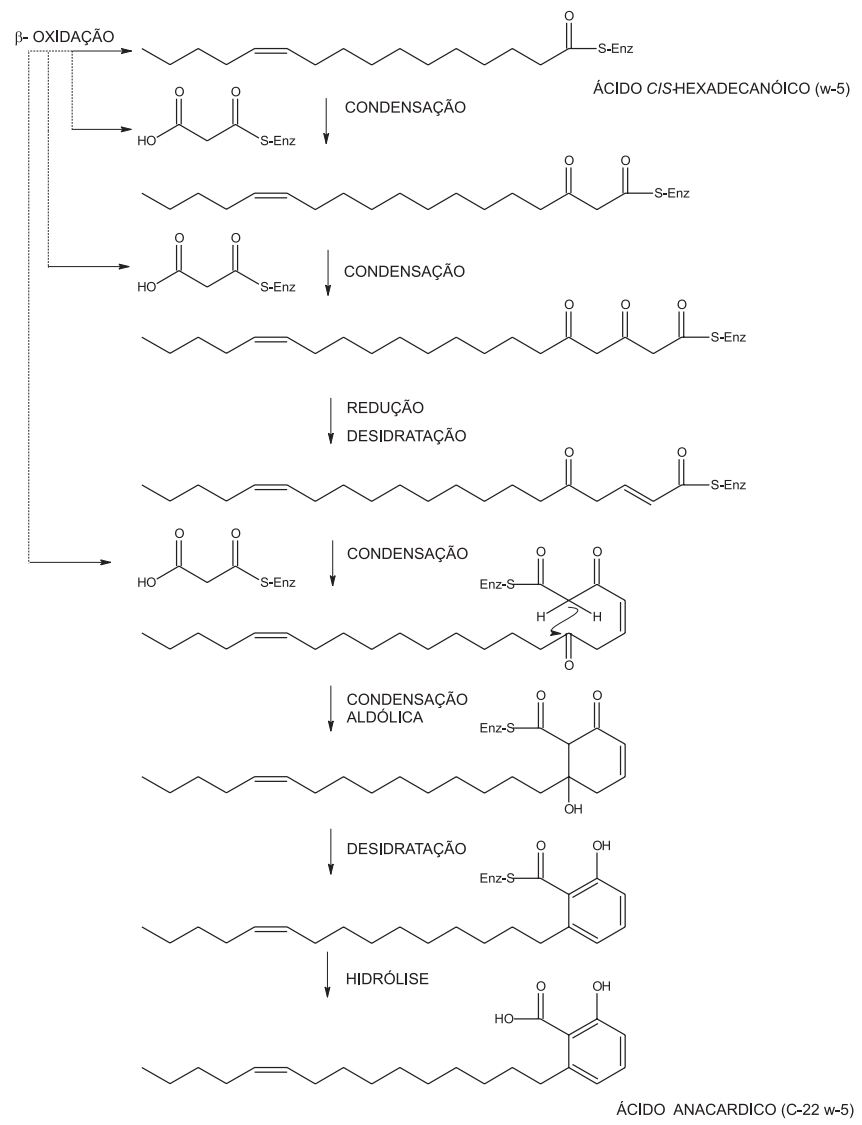

Figura 2. Proposta biossintética para os ácidos anacárdicos a partir de ácidos graxos. Adaptada da ref. 44

\section{Lipídios fenólicos não usuais na família Anacardiaceae}

Em espécies dos gêneros Tapirira e Lannea foi observada a 
ocorrência de lipídios fenólicos não usuais à família. O gênero Tapirira é composto de aproximadamente 15 espécies, que ocorrem principalmente na América do Sul. Nos últimos anos, de Tapirira foram isolados derivados alquilados da ciclo-hexanona (265 e 271 $)^{85}$, que parecem ser possíveis precursores dos lipídios fenólicos. $\mathrm{O}$ isolamento dessas substâncias é mais um dado que corrobora a proposta biossintética de Walters e colaboradores ${ }^{87}$. Estes derivados alquilados da ciclo-hexanona também já foram relatados em duas espécies do gênero Lannea, L. welwitschii e $L$. edulis $^{73,77}$ (Tabela 3).

Além dos possíveis precursores dos lipídios fenólicos, de Tapirira foram obtidos derivados $\omega$-fenilalquílico da ciclo-hexanona, bem como $\omega$-fenilalquilfenóis. Os compostos fenólicos substituídos com cadeia longa $\omega$-fenílalquílica foram isolados pela primeira vez de Melanorrhoea usitate (Anacardiaceae) ${ }^{80}$. Os ácidos cinâmicos e benzóico são os possíveis precursores do grupo $\omega$ fenil na cadeia lateral das referidas substâncias. Deste modo, foi sugerido que os $\omega$-fenilalquilfenóis (204-208, 222, 224-225, 267 270) são derivados de duas rotas biossintéticas consecutivas, a do ácido chiquímico e a dos policetídeos ${ }^{80}$ (Figura 3), diferentemente do que se poderia esperar se comparados com os lipídios fenólicos, sem a participação dos ácidos graxos.

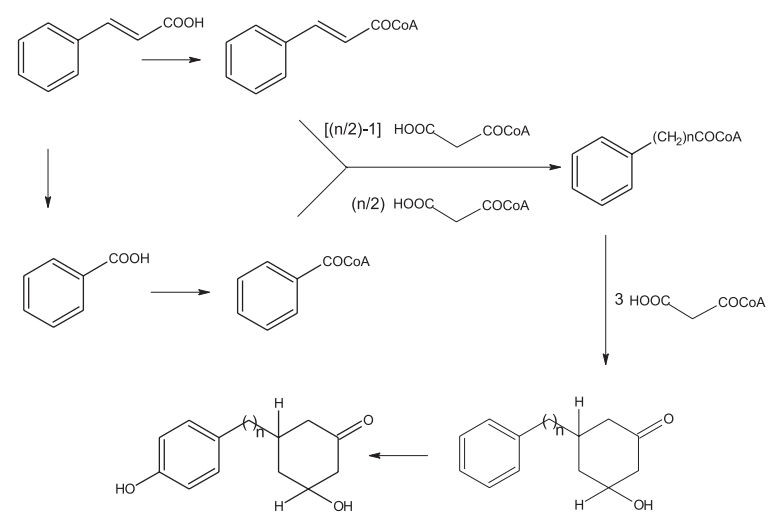

Figura 3. Proposta biossintética para $\omega$-fenil-alquilfenóis e derivados. Adaptada da ref 52

\section{BENZENÓIDES DE ANACARDIACEAE}

Além dos triterpenos, biflavonóides e lipídeos fenólicos, que são as substâncias de maior ocorrência nas espécies da família, também se encontram relatos do isolamento de outras classes de substâncias diferentes (Tabela 4). Uma vez mais, o exemplo importante é a xantona glicosilada conhecida como mangiferina, que é o principal componente da formulação farmacêutica Vimang ${ }^{\circledR}$. Outras substâncias que ocorrem em espécies da família caracterizam-se pela simplicidade estrutural de derivados de fenóis e ácidos cinâmicos. Apesar de existir somente um estudo químico relacionado com espécies do gênero Dracontomelum, o isolamento de 275 é o único relato de ocorrência de alcalóides na família.

\section{CONSIDERAÇÕES FINAIS}

As espécies de Anacardiaceae mais estudadas do ponto de vista químico têm sido Mangifera indica, Anacardium occidentalle e algumas espécies de Rhus, provavelmente devido à importância econômica e toxicidade demonstrada pelas espécies da família. Deve-se salientar, entretanto, que estes estudos com as espécies citadas representam menos de $10 \%$ das espécies descritas para estes gêneros. Mesmo do ponto de vista geral, menos de $7 \%$ das
Tabela 4. Benzenóides isolados de espécies de Anacardiaceae

$\begin{array}{cc}\text { Cotinus coggygria }^{47} \\ 272 & 273: \mathrm{R}=\mathrm{H} \\ & 274: \mathrm{CH}_{3}\end{array}$

Dracontomelum
mangiferum ${ }^{89}$

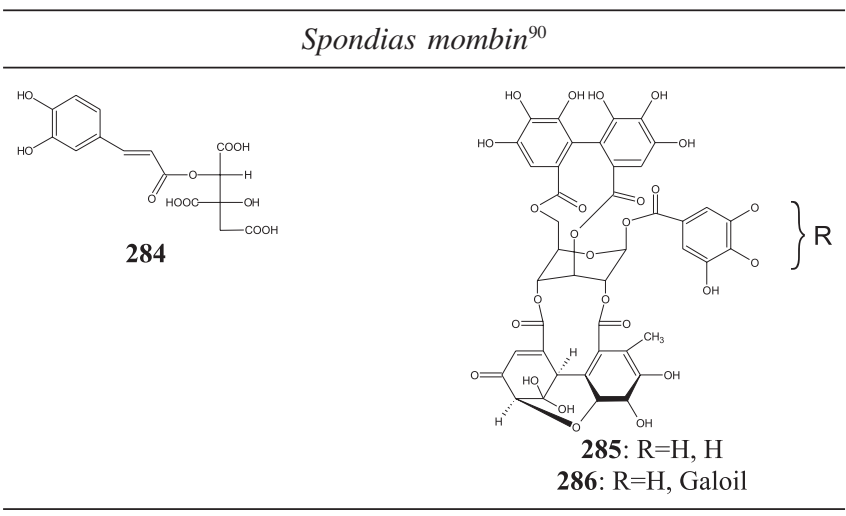

Tapirira guianensi ${ }^{2}$

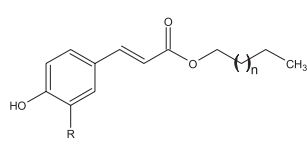

287: $\mathrm{R}=\mathrm{OMe} ; \mathrm{n}=20$

288: $\mathrm{R}=\mathrm{OMe} ; \mathrm{n}=22$

289: $\mathrm{R}=\mathrm{OMe} ; \mathrm{n}=24$

290: $\mathrm{R}=\mathrm{OMe} ; \mathrm{n}=26$

291: $\mathrm{R}=\mathrm{OMe} ; \mathrm{n}=28$

292: $\mathrm{R}=\mathrm{H} ; \mathrm{n}=17$ 
espécies conhecidas da família tiveram estudos fitoquímico e de atividade já realizados. Nesses poucos estudos pode-se constatar que duas classes de substâncias são características na família: flavonóides, especialmente biflavonóides, e lipídios fenólicos, encontrados em espécies que normalmente apresentam propriedades tóxicas ou alergênicas. A diversidade de metabólitos e atividades biológicas têm justificado o enorme interesse no estudo de espécies desta família. A atividade antioxidante ampla e elevada apresentada pelo Vimang ${ }^{\circledR}$ está entre os resultados mais promissores até o momento. Os lipídeos fenólicos, derivados da rota policetídica, são metabólitos freqüientes em espécies desta família e muitas atividades biológicas relatadas e comprovadas destas espécies são atribuídas a esta classe de substâncias. Pesquisas com espécies ainda não estudadas têm possibilitado a comprovação das propostas biossintéticas dos lipídios fenólicos, bem como registrar a ocorrência de substâncias não usuais e de elevada atividade biológica.

\section{AGRADECIMENTOS}

Ao CNPq e à CAPES pelas bolsas de estudo e à FAPESB, ao $\mathrm{CNPq}$ e ao Instituto do Milênio do Semi-Árido (IMSEAR/CNPq/ MCT) pelos auxílios financeiros.

\section{REFERENCIAS}

1. Vogl, O.; Mitchell, J. D.; Pure Appl. Chem. 1996, 33, 1581.

2. Vogl, O.; Qin, M.; Mitchell, J. D.; Cellul. Chem. Technol. 1995, 29, 273.

3. Evans, F. J.; Schmidt, R. J.; Planta Med. 1980, 38, 289.

4. Tyman, J. H. P.; Chem. Soc. Rev. 1979, 8, 499.

5. Eiadthong, W.; Yonemori, K.; Sugiura, A.; Utsunomiya, N.; Subhadrabandhu, S.; Scientia Hortic. 1999, 80, 145.

6. Garrido, G.; González, D.; Lemus, Y.; García, D.; Lodeiro, L.; Quintero, G.; Delporte, C.; Núnes-Sellés, A. J.; Delgado, R.; Pharmacol. Res. 2004, $50,143$.

7. Makare, N.; Bodhankar, S.; Rangari, V.; J. Ethnopharmacol. 2001, 78, 133.

8. Sanchez, G. M.; Re, L. A.; Giuliani, A.; Núnez-Sellés, J.; Davison, G. P.; León-Fernández, O. S.; Pharmacol. Res. 2000, 42, 6.

9. Muruganandan, S.; Gupta, S.; Kataria, M.; Lal, J.; Gupta, P. K.; Toxicology 2002, 176, 165

10. Selles, A. J. N.; Castro, H. T.; Aguëro-Aguëro, V. J.; González-González, J.; Naddeo, F.; De Simone; F.; Rastrelli, L.; J. Agric. Food Chem. 2002, $50,762$.

11. Beltrán, A. E.; Alvarez, Y.; Xavier, F. E.; Herranz, R.; Rodríguez, J.; Núnez, A. J.; Alonso, M. J.; Salaices, M.; Eur. J. Pharmacol. 2004, 499, 297.

12. Anjaneyulu, V.; Satyanarayana, P.; Viswanadham, K. N.; Jyothi, V. G.; Rao, K. N.; Radhika, P.; Phytochemistry 1999, 50, 1229.

13. Bandyopadhyay, C.; Gholap, A. S.; Mamdapur, V. R.; J. Agric. Food Chem. 1985, 33, 377.

14. Sharma, S. K.; Ali, M.; J. Indian Chem. Soc. 1995, 72, 339; Khan, M. N. I.; Nizami, S. S.; Khan, M. A.; J. Nat. Prod. 1993, 56, 767; Khan, M. A.; Nizami, S. S.; Khan, M. N. I.; Azeem, S. W.; Ahamed, Z.; J. Nat. Prod. 1994, 57, 988; Anjaneyulu, V.; Radica, P.; Indian J. Chem., Sect B: Org. Chem. Incl. Med. Chem. 2000, 39, 883.

15. Si, X.; Wei, S.; Xu, X.; Fang, X.; Wu, W.; Chin. J. Chin. Mater. Med. 1995, $20,295$.

16. Anjaneyulu, V.; Krishna, M. M.; Babu, J. S.; Babu, B. H.; Acta Cienc. Indica Chem. 1991, 17c, 395. (CA 117:44568e).

17. Khan, M. A.; Nizami, S. S.; Khan, M. N. I.; Azeem, S. W.; Ahamed, Z.; J. Nat. Prod. 1994, 57, 988.

18. Marner, F.-J.; Freyer, A.; Lex, J.; Phytochemistry 1991, 30, 3709.

19. Caputo, R.; Mangoni, L.; Monaco, P.; Palumbo, G.; Aynehchi, Y.; Bagheri, M.; Phytochemistry 1978, 17, 815.

20. Monaco, P.; Caputo, R.; Palumbo, G.; Mangoni, L.; Phytochemistry 1973, $12,939$.

21. Kuo, S.-C.; Teng, C.-M.; Lee, L.-G.; Chiu, T.-H.; Wu, T.-S.; Huang, S.-C.; Wu, J.-B.; Shieh, T.-Y.; Chang, R.-J.; Chou, T.-C.; Planta Med. 1991, 247.

22. Yürüker, A.; Orjala, J.; Sticher, O.; Rali, T.; Phytochemistry 1998, 48, 863

23. Schmidt, J.; Porzel, A.; Adam, G.; Phytochemistry 1998, 49, 2049.

24. Cardoso, M. P.; David, J. M.; David, J. P.; Nat. Prod. Res. 2005, 19, 431.

25. Campello, J. de P.; Marsaioli, A. J.; Phytochemistry 1975, 14, 2300.

26. Pozzo-Balbi, T.; Nobile, L.; Scarpini, G.; Cini, M.; Gazz. Chim. Ital. 1976, 106, 785 (CA 86:140290v); Terhune, S.; Hogg, J. W.; Lawrence, B. M.; Phytochemistry 1974, 13, 865.
27. Singh, R. B.; Saxena, V. K.; J. Inst. Chem. 1976, 48, 299. (CA 87:18994h). 28. Tandon, S.; Rastogi, R. P.; Planta Med. 1976, 29, 190.

29. Correia, S. de J.; David, J. P.; David, J. M.; Quim. Nova. 2003, 26, 36.

30. Lin, Y. M.; Chem, F. C.; Phytochemistry 1974, 13, 658.

31. Lin, Y. M.; Chem, F. C.; Lee, K. H.; Planta Med. 1989, 55, 166

32. Lin, Y. M.; Chem, F. C.; Phytochemistry 1974, 13, 1617.

33. Lin, Y. M.; Anderson, H.; Flavin, M. T.; Pai, Y. H. S.; J. Nat. Prod. 1997, 60,884

34. Mesesane, I. B.; Yeboah, S. O.; Liebscher, J.; Mügge, C.; Abegaz, B. M.; Phytochemistry 2000, 53, 1005.

35. Ahamed, M. S.; Galal, A. M.; Ross, S. A.; Ferreira, D.; Elsohly, M. A.; Ibrahim, A. R. S.; Mossa, J. S.; El-Feraly, F. S.; Phytochemistry 2001, 58, 599.

36. Zembower, D. E.; Lin, Y.; Flavin, M. T.; Chen, F.; Korba, B. E.; Antiviral Res. 1998, 39, 81; Lin, Y.M.; Zembower, D. E.; Flavin, M. T.; Schure, R. M.; Anderson, H. M.; Korba, B. E.; Chen, F.-C.; Bioorg. Med. Chem. Lett. 1997, 7, 2325.

37. Parveen, M.; Khan, N. U.; Curr. Sci. 1987, 56, 1171.

38. Van Loo, P.; De Bruyn, A.; Verzele, M.; Chromatographia 1988, 25, 15.

39. Wollenweber, E.; Z. Naturforsch., C: J. Biosci. 1974, 29, 52.

40. Nair, A. G. R.; Kotiyal, J. P.; Bhardwaj, D. K.; Phytochemistry 1983, 22, 318.

41. Mdee, L. K.; Yeboah, S. O.; Abegaz, B. M.; J. Nat. Prod. 2003, 66, 599.

42. Shobha, S. V.; Krishnaswamy, P. R.; Ravindranath, B.; Phytochemistry 1992, 31, 2295.

43. Kubo, I.; Muroi, H.; Kubo, A.; J. Nat. Prod. 1994, 57, 9.

44. Arya, R.; Ilyas, M.; Nasim, K. T.; Phytochemistry 1992, 31, 2569.

45. Weniger, B.; Vonthron-Senecheau, C; Arango, G. J.; Kaiser, M.; Brun, R.; Anton, R.; Fitoterapia 2004, 75, 764.

46. Phan, T. S.; Nguyen, V. D.; Le T. H.; Nguyen, H. K.; Tap. Chi. Hoa. Hoc. 1993, 31, 76 (CA 122:235279z)

47. Westenburg, H. E.; Lee, K.-J.; Lee, S. K.; Fong, H. H. S.; van Breemen, R. B.; Pezzuto, J. M.; Kinghorn, A. D.; J. Nat. Prod. 2000, 63, 1696.

48. Sultana, S.; Ilyas, M.; Phytochemistry 1986, 25, 963.

49. Islam, M. T.; Tahara, S.; Phytochemistry 2000, 54, 901; Subramanian, S. S.; Nair, A. G. R.; Phytochemistry 1971, 10, 1939.

50. Viana, G. S. B.; Bandeira, M. A. M.; Matos, F. J. A.; Phytomedicine 2003, $10,189$.

51. Imamura, H.; Ohta, H.; Kiriyama, S.; Ohashi, H. I.; Gifu Daigaku Nogakubu Kenkyu Hokoku 1979, 42, 117 (CA 93:22611x).

52. Braca, A.; Politi, M.; Sanogo, R.; Sanou, H.; Morelli, I.; Pizza, C.; Tommasi de, N.; J. Agric. Food Chem. 2003, 51, 6689.

53. Ahamad, I.; Ishratullah, K.; Ilyas, M.; Rahman, W.; Seligmann, O.; Wagner, H.; Phytochemistry 1981, 20, 1169

54. Murthy, S. S. N.; Phytochemistry 1985, 24, 1065; Gil, R. R.; Lin, L-Z; Cordell, G. A.; Kumar, M. R.; Ramesh, M.; Reddy, B. M.; Mohan, G. K.; Rao, A. V. N. A.; Phytochemistry 1995, 39, 405; Murthy, S. S. N.; Phytochemistry 1988, 27, 3020; Murthy, S. S. N.; Proc. Indian Acad. Sci. 1986, 97, 63

55. Kumanotani, J.; Prog. Org. Coat. 1995, 26, 163.

56. Wu, P.-L.; Lin, S.-B.; Huang, C.-P.; Chiou, R. Y.-Y.; J. Nat. Prod. 2002, $65,1719$.

57. Goetz, G. M.; Lepoittevin, J. P.; Bioorg. Med. Chem. Lett. 1999, 9, 1141.

58. Dos Santos, M. L.; De Magalhães, G. C.; J. Braz. Chem. Soc. 1999, 10, 13

59. Agostini-Costa, T. S.; Jales, K. A.; Garruti, D. S.; Padilha, V. A.; De Lima, J. B.; Aguiar, M. J.; Paiva, J. R.; Ciência Rural 2004, 34, 1075.

60. Prithiviraj, B.; Manickam, M.; Singh, U. P.; Ray, A. B.; Can. J. Bot. 1997, 75, 207.

61. Bouillant, M. L.; Jacoud, C.; Zanella, I.; Favre-Bonvin, J.; Bally, R.; Phytochemistry 1994, 35, 769 .

62. Deszcz, L.; Kozubek, A.; Biochim. Biophys. Acta 2000, 1483, 241.

63. Kubo, I.; Kinst-Hori, I.; Yokokawa, Y.; J. Nat. Prod. 1994, 57, 545.

64. Paramashivappa, R.; Kumar, P. P.; Vithayathil, P. J.; Rao, A. S.; J. Agric. Food Chem. 2001, 49, 2548

65. Kubo, I.; Muroi, H.; Himejima, M.; J. Agric. Food Chem. 1993, 41, 1016.

66. Kumar, K. P. V.; Sethuraman, M. G.; Prog. Org. Coat. 2004, 49, 244.

67. Sullivan, J. T.; Richards, C. S.; Lloyd, H. A.; Krishna, G.; Planta Med. 1982, 44, 175.

68. Kozubek, A.; Tyman, J. H. P.; Chem. Rev. 1999, 99, 1.

69. Yalpani, M.; Tyman, J. H. P.; Phytochemistry 1983, 10, 2263

70. Gambaro, V.; Chamy, M. C.; Von Brand, E.; Garbarino, J. A.; Planta Med. 1986, $1,20$.

71. Rivero-Cruz, J. F.; Chavez, D.; Bautista, B. H.; Anaya, A. L.; Mata, R.; Phytochemistry 1997, 45, 1003.

72. Reffstrup, T.; Boll, P. M.; Phytochemistry 1985, 24, 2563; Canedo, L. M.; Delcorral, J. M. M.; Sanfeliciano, A.; Phytochemistry 1997, 44, 1559. 
73. Groweiss, A.; Cardellina Ii, J. H.; Pannell, L. K.; Uyakul, D.; Kashaman, Y.; Boyd, M.; J. Nat. Prod. 1997, 60, 116.

74. David, J. M.; Chavez, J. P.; Chai, H. -B.; Pezzuto, J. M.; Cordell, G. A.; J. Nat. Prod. 1998, 61, 287.

75. Metzger, P.; Casadevall, E.; Phytochemistry 1989, 28, 2097.

76. Bhunia, H. P.; Nando, G. B.; Baasak, A.; Lenka, S.; Nayak, P. L.; Eur. Polym. J. 1999, 35, 1713.

77. Queiroz, E. F.; Kuhl, C.; Terreaux, C.; Mavi, S.; Hostettmann, K.; J. Nat. Prod. 2003, 66, 578.

78. Lopéz, P.; Ruffa, M. J.; Cavallaro, L.; Campos, R.; Ferraro, G.; Phytomedicine 2005, 12, 108.

79. Valcic, S.; Wächter, G. A.; Eppler. C. M.; Timmermann, B. N.; J. Nat. Prod. 2002, 65, 1270 .

80. Du, Y.; Oshima, R.; Yamanotani, J.; Miyakoshi, T.; Phytochemistry 1986, $25,2211$.

81. Adawadkar, P. D.; Elsohly, M. A.; Phytochemistry 1983, 22, 1280.

82. Carpenter, R. C.; Sotheeswaran, S.; Sultanbawa, M. U. S.; Balasubramaniam, S.; Phytochemistry 1980, 19, 445.
83. Rao, N. S. P.; Row, R.; Phytochemistry 1973, 12, 671.

84. Coates, N. J.; Gilpin, M. L.; Gwynn, M. N.; Lewis, D. E.; Milner, P. H.; Spear, S. R.; Tyler, J. W.; J. Nat. Prod. 1994, 57, 4

85. Correia, S. De J.; David, J. M.; David, J. P.; Chai, H.-B.; Pezzuto, J. M.; Cordell, G. A.; Phytochemistry 2001, 56, 781.

86. Birch, A. J.; Science 1967, 156, 202; Staunton, J.; Weissman, K. J.; Nat Prod. Rep. 2001, 18, 380.

87. Walters, D. S.; Craig, R.; Mumma, R. O.; Phytochemistry 1990, 29, 1815.

88. Dimroth, P.; Ringelmann, E.; Lynen, F.; Eur. J. Biochem. 1976, 68, 591; Dimroth, P.; Walter, H.; Lynen, F.; Eur. J. Biochem. 1970, 13, 98.

89. Johns, S. R.; Lamberto, J. A.; Occolowi, J. L.; Aust. J. Chem. 1966, 19 , 1951.

90. Corthout, J.; Pieters, L.; Claeys, M.; Berghe, D. V.; Vlietinck, A.; Phytochemistry 1992, 31, 1979; Corthout, J.; Pieters, L. A.; Claeys, M.; Berghe, D. A. V.; Vlietinck, A. J.; Phytochemistry 1991, 30, 1129. 